\title{
Object salience and code separation in picture naming
}

\author{
ROY LACHMAN, JANET L. LACHMAN, and CARROLL THRONESBERY \\ University of Houston, Houston, Texas 77004
}

and

LINDA S. SALA

Wayne State University, Detroit, Michigan 48202

\begin{abstract}
Picture naming requires access to three kinds of codes in permanent memory: visualperceptual, semantic, and lexical. When a picture is named a second time, there is a substantial latency drop. This is assumed to reflect a limited but specific reorganization of memory called "update." Research was designed to explore three questions: (1) Are the code systems independent? (2) Does update occur in the visual system? If so, what proportion of update is visual? (3) How does the salience of objects (i.e., frequency, ecological importance) determine the magnitude of the latency drop? The naming latencies for pictures previously processed only visually were compared with pictures renamed and those not previously presented. Pictures were calibrated on one index of salience (uncertainty). Results supported the conclusion that the visual system is at least partially independent of the others. Update occurs in the visual system; visual-node priming may be responsible for approximately one-third of the total latency drop. Finally, visual update appears insensitive to object salience, whereas the nonvisual components primed, lexical and/or semantic, interact with salience.
\end{abstract}

The concept of coding in cognitive psychology refers to the rules by which experience is converted into internal representations. A code, therefore, is the format of information that is being processed or that has previously been stored in memory. Three major types of code are typically described in the literature: physical or perceptual codes, name or lexical codes, and semantic codes. Posner (1978) in reviewing a massive literature, has made a compelling case for the three-code approach as opposed to single-code theory.

Ancillary theoretical mechanisms are required to represent each type of code in a permanent memory system and to convey convertibility relations between the code types. Information processing theories of memory often use network structures to represent internal storage of information (e.g., Anderson, 1976). In such theories, concepts are represented as nodes, and the total set of interconnections among specific nodes make up a graph network. Each node in a network must represent some element of information, such as a semantic concept, a concept name, or a sensory feature of a concept. Moreover, each code type must be represented by a unique type of node. The tripartite classification of codes, therefore, provides a minimal classification scheme for memory nodes, visual, semantic, and lexical, with several kinds of communication capability between them. The three sets may be so intimately linked that activating one kind of node invariably produces maximum activation of the associated others. Or they may be relatively independent, so that manipulations can be devised to activate, for example, only a visual node, without affecting the corresponding semantic and lexical nodes. Or the degree of independence may fall somewhere in between. A major purpose of this study was to determine if perceptual nodes can be separated, even partially, from linked semantic and lexical nodes.

The experimental task used for this investigation is picture naming, which implicates all three node types. In picture naming, a slide is projected, and a person names it under speeded conditions. Typical objects presented are table, chair, pagoda, and gyroscope. Naming latency data have been used to establish aspects of the organization of lexical memory and to determine major vectors of permanent memory (Carroll \& White, 1973; Lachman \& Lachman, 1980). A key fact about picture naming is that it produces powerful priming effects; when a picture is named a second time, latencies drop considerably, as much as several seconds. Priming has been widely studied under the rubric of activation, a short-lived process (Anderson, 1976). In picture naming, the facilitatory effect of a prior naming is typically of a greater magnitude than activation and is still detectable after 2 weeks (Lachman \& Lachman, 1980). To distinguish this durable priming effect from activation, we have chosen to call it the "update effect." Update may reflect a reordering of priorities within the visual, semantic, and/or lexical networks in order to deal efficiently with objects or words that have become topically more current. A word for a particular concept that has already occurred once in speech is likelier to be needed again soon. The update effect may be one mechanism mediating the speed and efficiency of word production in fluent speech. Over protracted periods of time (e.g., weeks or even years), the update effect may reflect an ecologically based adaptation that supports the efficient use of memory. Any information retrieval system can 
be organized to allocate its resources more or less efficiently. In an environment in which some information must be accessed frequently and other information only rarely, an efficient biological retrieval system would be organized in such a way as to retrieve frequently needed and currently required information most rapidly. Thus, more processing resources should be allocated to information that is salient-that is, needed most often. Update enables such a system to respond appropriately to salience and changes in salience. If prior use of an item of information increases its accessiblity, frequently needed material will become relatively more accessible and seldom needed material, relatively less accessible.

The concept of "ecological salience" is in the process of theoretical development (Lachman \& Lachman, 1980). Nevertheless, it is useful in interpreting the variables that have emerged as major vectors determining speed of retrieval from permanent memory. In picture naming, these variables are word frequency (Oldfield \& Wingfield, 1965), age of acquisition (Carrol \& White, 1973), and codability as measured by "Shannon" uncertainty (Lachman, Shaffer, and Hennrikus, 1974). Average uncertainty, or codability, reflects the diversity of names given to an object. The effects of the three variables on naming latency are independent, but highly correlated (Lachman et al., 1974). All can be considered to reflect aspects of an object's ecological salience. Salient objects have frequently encountered, early-learned, and readily agreedupon names. Some objects and their names, those that are encountered every day, are continually being "updated." These will be relatively insensitive to an additional update experience occurring in a laboratory experiment. Other objects and names, rarely encountered, may be substantially updated by one such experience. Indeed, using uncertainty as an estimate of salience, Lachman et al. (1974) found an interaction between uncertainty and update, reflecting a substantially greater latency drop on the second naming for items of high uncertainty. We employ normative uncertainty as an estimate of salience. Both frequency and acquisition age must be estimated from a single name word, usually the word that is most often given as the name of a picture. Such estimates can be misleading when a picture elicits a large number of different names; in such cases, the word used to estimate frequency or acquisition age may be given by a minority of subjects.

All theories of picture naming assume that this task, and object naming in general, requires two major processing stages (Clark \& Clark, 1977). Stage 1, consisting of the perceptual identification of the to-be-named object, is best described as essentially parallel and relatively fast. Stage 2 , semantic and lexical access, is thought to involve a search through an internal lexicon and to be relatively slow, depending on the magnitude of the search. This analysis would suggest that updating occurs primarily in processes associated with Stage 2, that is, in semantic and/or lexical searches as opposed to visual or perceptual processing. A variety of laboratory tasks have yielded data that support this conclusion. Because the magnitude of update increases with uncertainty/salience, it appears that the update effect must reside in an uncertainty-sensitive process. Visual processing, however, does not show uncertainty effects (Lachman \& Lachman, 1980; Mills, Knox, Juola, \& Salmon, 1979). Matching two versions of a picture, without naming, yields reaction times with approximately a zero slope over uncertainty. This matching process, then, while it clearly requires Stage 1 visual processing, is independent of uncertainty. These arguments seem to exclude the visual system as a likely locus of update effects. Furthermore, the latency to match a picture to a property (e.g., a picture of a ham sandwich to the property "is edible") is also insensitive to uncertainty (Lachman \& Lachman, 1980).

Bartram (1974), on the other hand, found outcomes that seem to indicate visual updating. He found that repeated naming produced progressive decrements in naming latencies-the typical update effect. However, a picture of the same object, shown at a different angle of view, produced a smaller update effect than did the identical picture. This pattern of outcomes suggests that some portion of the latency decrement is attributable to update of the visual system. Obviously, if it requires an identical picture on two naming occasions to maximize the update effect, Stage 1 (visual processing) would appear to be implicated. The reasoning in both cases is somewhat indirect; however, the conclusions conflict. Bartram's results support the conclusion that update effects do occur in the visual system; the Lachman and Lachman (1980) and Mills et al. (1979) data suggest the opposite.

Subjects overtly produced object names in all treatments of Bartram's (1974) study. The ideal experiment to resolve the issue would be one in which the effect of "pure" visual processing on subsequent naming can be assessed. In the present study, we use a mock recognition test and capitalize on the subject's belief that he is only to decide if a picture has been seen before. There is no apparent incentive, here, to engage in semantic analysis or to produce the name of the object. The experiment was designed to present some pictures in a standard naming condition and others as "foils" in a recognition task and to assess the effect of such prior processing on subsequent naming.

\section{METHOD}

\section{Design}

Sums of squares and expected mean squares were obtained from BMD08V for calculating quasi Fs. The variables and levels were subjects (three), counterbalance (six), treatments (three), uncertainty (seven), and picture slides (seven). Pictures and subjects were treated as random effects; treatments, uncertainty, and counterbalancing were treated as fixed effects. Pictures were nested within uncertainty, and subjects were nested within counterbalancing. Thus, three subjects were assigned to each of six counterbalanced orders of pictures within treatments, for a total of 18 subjects. Seven pictures for each of seven levels of uncertainty were assigned to each of three treatments, for a total of 147 picture slides. The three treatments were prior naming, prior visual exposure, and no prior exposure. 
Subjects

Subjects were 18 female students from an introductory psychology course.

\section{Stimuli and Uncertainty}

The basic stimulus set consisted of 147 slides of color photographs that depicted objects or visual configurations that could be named with one word. Uncertainty values for the stimuli had previously been calculated from the naming responses of an independent normative sample. The procedure used for the determination of picture uncertainty has been described in detail elsewhere (Lachman et al., 1974). On the basis of these normative data, the stimuli were selected so that they divided into seven sets of 21 , with median uncertainty per set approximately equal to $.0, .5,1.0,1.5,2.0,2.5$, and 3.0 bits. The uncertainty value is highly correlated with the number of different names elicited by a picture.

In addition to the 147-slide basic stimulus set, 26 slides were used as practice stimuli. Another 49 slides were first named, then used as "old" stimuli in a recognition task, and then discarded. These stimuli were slides depicting nameable visual configurations that were not in the basic set, but that ranged roughly over the same uncertainty levels as the basic set.

\section{Apparatus}

A solid state shutter was positioned in front of the lens of a Carousel slide projector that was wired to various gates and timers. When the subject pressed a button on the front panel, the shutter opened and a digital clock began recording elapsed time. The subject's vocal response into a boom microphone activated a voice-operated relay that stopped the clock, closed the shutter, initiated a 4-sec intertrial interval, and advanced the projector. The experimenter recorded each response name and response latency and, when ready for the next stimulus presentation, pressed a button. A logical "and" condition, consisting of the experimenter's buttonpress and completion of the 4-sec interval, illuminated a red light in front of the subject, indicating that the system was ready for another stimulus presentation. The subject, when ready, pressed the panel button to initiate the next slide cycle. Slides were projected onto a rear-viewing screen; the projected picture was $20 \times 29 \mathrm{~cm}$. The screen was approximately $55 \mathrm{~cm}$ from the subject at eye level.

\section{Phase 1}

During this phase, the subjects performed the standard naming task. The subject was instructed to fix her eyes on the center of the screen when the "system ready" light came on and to press the "start" button when ready. She was told to respond as accurately and as quickly as possible with a single-word name for the projected picture. Sixteen practice slides were shown; feedback on appropriateness of response was given for these slides. If the subject responded to the picture of a man wearing a turban with "man," for example, "Hindu" was suggested as a more specific response. The subject was then presented 98 slides to name. Of these, 49 were those in the renaming subset; they would appear in Phase 3 to be named again. The other 49 would be shown in Phase 2 as "old" items in a recognition test and then discarded.

\section{Phase 2}

Phase 2 consisted of the recognition test. The subject was informed that we were testing how well she could recognize the slides that she had just seen. She was asked to say "yes" if she recognized a slide as having been shown during Phase 1 and "no" if it was a new slide that she did not recognize from Phase 1 ; the spoken response stopped the clock. The subject was shown a set of 10 practice slides, consisting of 5 previously used practice slides and 5 new ones. Then the 49 "throw-away" slides named in Phase 1 were presented, intermixed with 49 "new" slides. These "new" slides were those assigned to the visual treatment; they would appear for naming in Phase 3. New-old classification was nearly $100 \%$ correct.
Table 1

Experimental Treatments

\begin{tabular}{llll}
\hline \multicolumn{1}{c}{ Treatment } & $\begin{array}{l}\text { Phase 1 } \\
\text { Naming }\end{array}$ & \multicolumn{1}{c}{$\begin{array}{c}\text { Phase 2 } \\
\text { Recognition }\end{array}$} & \multicolumn{1}{c}{ Phase 3 } \\
Naming
\end{tabular}

*Stimuli not used in this phase.

\section{Phase 3}

Phase 3 was the final phase of the experiment and consisted of a standard naming task. Several previous studies found no differences between variable slide durations determined by the subject's naming latency and a fixed duration of 3 or $5 \mathrm{sec}$ (Lachman \& Lachman, 1980). The stimuli were three subsets of 49 slides each, corresponding to the three experimental treatments. The 49 slides that had been named in Phase 1 and excluded from Phase 2 constituted the "name-rename" treatment. The 49 slides that had been presented as "new" items in the recognition task constituted the "visual" treatment. There were also 49 slides that had not been presented previously in the experiment; these constituted the "no prior exposure" treatment. Table 1 illustrates each condition.

\section{RESULTS AND DISCUSSION}

\section{Errors}

There was no speed-accuracy tradeoff in picture naming. Response faults covaried with latency. The most obvious kind of error was failure to name a picture; this occurred only at high uncertainties. Of 18 subjects, 10 had no failure to name, 7 had one failure, and 1 subject had three failures. Some names were difficult to classify as "correct" or "incorrect" (e.g., gears named "watch"). Others reflected the subject's effort to find the name and, after a long latency, settling for a related name (e.g., cicada named "fly"). In all cases, response faults occurred with long reaction times.

\section{Latencies}

The data of interest in this experiment were the naming reaction times of the final naming phase. Both subjects and stimuli were treated as random variables in ANOVA. The data are plotted in Figure 1. Treatment was significant $\left[F^{\prime}(2,20.8)=26.487, p<.001\right]$. Uncertainty was also significant $\left[\mathrm{F}^{\prime}(6,60.1)=30.521\right.$, $\mathrm{p}<.001]$, as was the Treatment by Uncertainty interaction $\left[\mathrm{F}^{\prime}(12,65.4)=3.792, \mathrm{p}<.001\right]$. The counterbalancing condition was not significant $\left[\mathrm{F}^{\prime}(5,12.4)<1\right]$ in this or any other analysis, and henceforth it will not be reported. The Treatment by Uncertainty interaction reflects the differential sensitivity of the visual and namerename conditions to uncertainty level. This can be interpreted to mean that visual update effects and the total update effects produced by naming twice were differentially responsive to levels of environmental salience. It appears that total update was influenced by salience, but visual update was not. The magnitude of visual update effects at different levels of salience can be estimated by subtracting reaction times in the visual condition from those for the no prior exposure condi- 


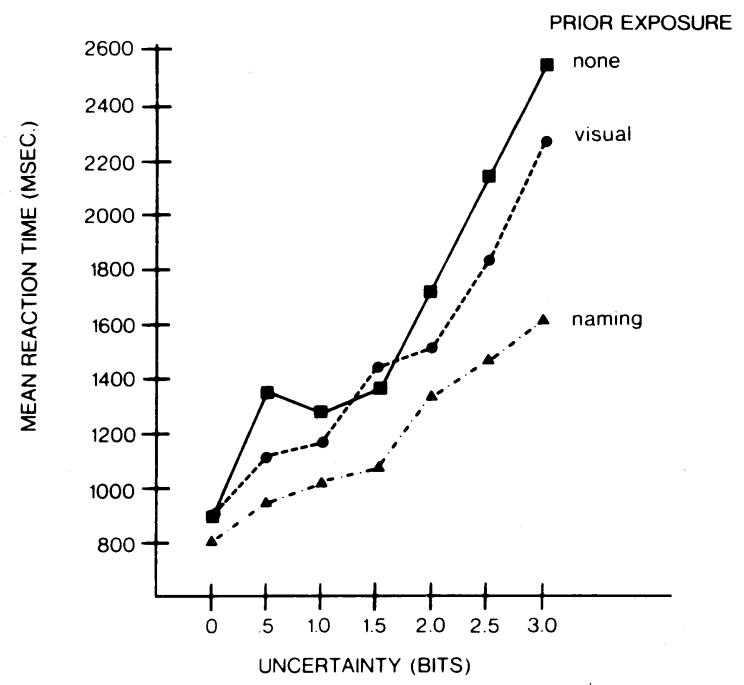

Figure 1. Naming latencies following naming, visual, or no prior exposure. Lexical uncertainty is a quantitative index of ecological salience.

tion at each level of uncertainty in Figure 1. The actual mean differences in milliseconds were $6,232,105,-63$, 224,336 , and 160 for $U=1, .5,1,1.5,2,2.5$, and 3 bits, respectively. These differences showed no systematic relationship to uncertainty; the best-fit line was essentially flat across uncertainty. In contrast, when the total update is estimated by subtracting reaction times in the name-rename condition from those in the no prior exposure condition, there was a strong effect of uncertainty, especially at $\mathrm{U}>2.0$ bits. The mean differences from $U=0$ to $U=3$ were $91,396,254,320,391,692$, and $962 \mathrm{msec}$. Thus, visual update may have a fixed value at all levels of uncertainty, but update may double and triple at $U=2.5$ and 3 bits.

If the three possible pairs of treatments are analyzed separately, the above interpretation implies predicted outcomes. The Treatment by Uncertainty interaction should be significant when the name-rename and visual treatments are compared and when the name-rename and no prior exposure treatments are compared. However, the interaction should not appear when the visual and no prior exposure treatments are compared. These three pairwise analyses were completed and the prediction outcomes obtained. For name-rename vs. visual, three $F^{\prime}$ ratios were significant $\left[F^{\prime}(1,10.2)=17.59\right.$, $\mathrm{p}<.005$, for treatments; $F^{\prime}(6,57.9)=23.17, p<.001$, for uncertainty; and $F^{\prime}(6,32.9)=3.17, p<.025$, for the interaction]. A similar outcome was obtained for namerename vs. no prior exposure $\left[\mathrm{F}^{\prime}(1,10.6)=58.07\right.$, $\mathrm{p}<.001$, for treatments; $\mathrm{F}^{\prime}(6,45.9)=28.66, \mathrm{p}<.001$, for uncertainty; and $F^{\prime}(6,31)=7.37, p<.001$, for the interaction]. When visual was compared with no prior exposure, $F^{\prime}(1,10.3)=7.41(\mathrm{p}<.025)$ for treatments and $F^{\prime}(6,61.5)=27.79(p<.001)$ for uncertainty; but, as expected, the Treatment by Uncertainty interaction was not significant $\left[F^{\prime}(6,33.7)=1.58\right]$. The reaction time data thus indicated that visual update occurs, but its magnitude is fixed and unrelated to salience as estimated by uncertainty. Total update apparently incorporates visual update and additional update components, some or all of which are salience determined. Further evidence that visual processing is independent of uncertainty came from reaction times collected during the recognition test. This task involved visual but not lexical processing and also generated a flat curve across uncertainty. The mean recognition latencies were $823,839,838,823,845,875$, and 832 $\mathrm{msec}$ for pictures with uncertainty levels of $0, .5,1,1.5$, $2,2.5$, and 3.0 bits.

\section{GENERAL CONCLUSIONS}

The results support the following answers to the questions initially posed. (1) There is some degree of independence between visual information stored in the brain and corresponding semantic or lexical information. (2) There is update in the visual system, consistent with Bartram's (1974) data. Prior naming produces an update effect on subsequent renaming, consisting of visual update plus one or more additional components. (3) The amount of update due to prior visual processing is approximately constant across levels of the ecological salience of the objects in the stimuli. The remaining update due to prior semantic and/or lexical processing is sensitive to levels of salience. The least salient objects show the greatest drop because their names (and meanings) are less frequently encountered. The names of the most salient objects $(U=0)$, in contrast, may be encountered weekly or even daily. They, therefore, are constantly updated, and their initial naming latencies in the laboratory are near the physiological lower limit.

\section{REFERENCES}

Anderson, J. R. Language, memory and thought. Hillsdale, N.J: Erlbaum, 1976.

Bartram, D. J. The role of visual and semantic codes in object naming. Cognitive Psychology, 1974, 6, 325-356.

Carroll, J. B., \& White, M. N. Word frequency and age of acquisition as determiners of picture-naming latency. Quarterly Journal of Experimental Psychology, 1973, 25, 85-95.

Clark, H. H., \& Clark, E. V. Psychology and language: An introduction to psycholinguistics. New York: Harcourt Brace Javanovich, 1977.

Lachman, R., \& Lachman, J. L. Picture naming: Retrieval and activation of long-term memory. In L. W. Poon, J. L. Fozard, L. S. Cermak, D. Arenberg, \& L. W. Thompson (Eds.), New directions in memory and aging: Proceedings of the George Talland memorial conference. Hilisdale, N.J: Erlbaum, 1980.

Lachman, R., Shaffer, J. P., \& Hennrikus, D. Language and cognition: Effects of stimulus codability, name-word frequency, and age of acquisition on lexical reaction time. Journal of Verbal Learning and Verbal Behavior, 1974, 13, 613-625.

Mills, R. H., Knox, A. W., Juola, J. F., \& Salmon, S. J. Cognitive loci of impairments in picture naming by aphasic subjects. Journal of Speech and Hearing Research, 1979, 22, 74-87.

OldField, R. C., \& Wingfield, A. Response latencies in naming objects. Quarterly Journal of Experimental Psychology, 1965, 17, 273-281.

Posner, M. I. Chronometric explorations of mind. The third Paul M. Fitts lectures. Hillsdale, N.J: Erlbaum, 1978.

(Received for publication June 21, 1980.) 